\title{
Surgical nursing care was viewed as engaged or detached by women in hospital for hip surgery
}

\author{
Kralik D, Koch T, Wotton K. Engagement and detachment: understanding patients'experiences with nursing.J Adv Nurs 1997 \\ Aug;26:399-407.
}

\section{Objective}

To describe patients' perceptions of the nursing care that they received after total hip replacement surgery.

\section{Design}

Phenomenology.

\section{Setting}

A large public, acute care hospital in South Australia.

\section{Patients}

9 women who were in hospital for a total hip replacement. 3 men who had hip surgery during the study declined to participate.

\section{Methods}

Over a 10 month period in 1995, the women were interviewed before surgery, after surgery, and after discharge. Data saturation occurred after multiple interviews with each of 9 participants. Each woman was asked to describe her experiences of the nursing care that she received, including what was important to her about the care she received and the way in which she felt the care had affected her. Interviews were tape recorded and then transcribed for further analysis.

\section{Main results}

2 major themes emerged. All women felt that they had come to the hospital with expectations about the care that they would receive. The presence of an engaged nurse (actively involved in care of the patient) was perceived to be a positive experience. Engaged nurses acknowledged the physical, emotional, spiritual, and environmental aspects of the patient and provided care that assisted recovery from surgery. Each patient was treated humanely and with compassion and the nurse projected a sensitivity that helped develop insight into each patient's situation. Minor themes related to engagement behaviour were expressed using the following phrases: nothing was too much trouble; asked or consulted me, or both; cheerful and humorous; compassionate and kind; knew what I wanted without asking; always available; gentle touch; and friendly and warm.

The care provided by a detached nurse was perceived as a negative experience. Detached nurses used a procedural approach to care and avoided personal contact with the patient which led to feelings of vulnerability and insecurity for the women. These nurses were considered authoritarian, uninterested in the patient as a person, and conveyed the expectation that the patient should be doing more for herself. Minor themes related to detachment behaviour were expressed using the following phrases: treated as a number or an object; too efficient and busy; made me feel I was lazy; you have to go along with whatever is happening to you and you feel uninformed; sharp or cold, or both, in approach; rough with physical care; just a job; and nurses did what they were told to do by other health professionals.

\section{Conclusions}

Women placed importance on the interaction between themselves and nurses during a hospital stay for surgery. The interaction was seen as positive (engaged) or negative (detached).

Source of funding: not stated.

For article reprint:D Kralik, 36 Beeston Way, West Lakes, South Australia, 5021 Australia. email Kralik@bigpond.com

\section{Commentary}

This study by Kralik et al offers a rich description of the relationship between nurse and patient. The methodology used is suitable to help uncover hidden interactions occurring at the bedside.

Previous studies have revealed similar findings. The authors of these studies reported that the patients experienced engagement when they felt cared for, were acknowledged as individuals, and when the nurse used humour, was available, and actively listened. ${ }^{12}$ Kralik et al described detachment when nurses approached the patients as objects, hurried and appeared too busy, did not communicate with the patients, did not involve patients in decisions, and handled the patients roughly.
On the theme of engagement it was reassuring to read about the positivity of the nurses who exhibited humour, openness, and warmth towards their patients. Where detachment was discussed, one became uneasy that patients were feeling that they had little or no say in their care and that the nursing care which they received may have made them feel worse.

The patients' experience of being nursed is important for nurses to understand. Before nurses can plan and implement care to meet the individual patient's needs, they must identify the patient's perceived needs and they must understand the way in which the patient views their nursing care. As we face decreased lengths of hospital stays, a focus on cost reduction, increased use of medical technology, and heavier nursing workloads, this study is timely in reminding us about the importance of "being with" patients, acknowledging their uniqueness as individuals, and including them in the planning and implementation of their care.

Christopher C Bassett, RN BA(Hons) Lecturer in Acute and Critical Care University of Sheffield South Yorkshire, UK

1 Taylor B. Being Human. Ordinariness in nursing. Melbourne: Churchill Livingstone, 1994

Koch T, Webb C, Williams A. Listening to the voices of older patients: an existentialphenomenological approach to quality assurance.J Clin Nurs 1995;7:8-10. 